\title{
Giant ethmoidal mucocele leading to proptosis and hypertelorism in a pediatric patient with cystic fibrosis: a case report
}

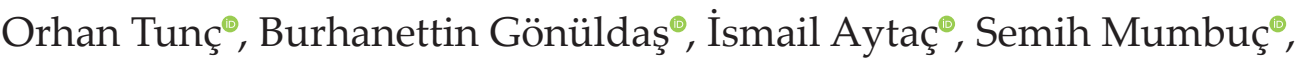 \\ Muzaffer Kanlıkama ${ }^{\circ}$ \\ Department of Otorhinolaryngology, Gaziantep University Faculty of Medicine, Gaziantep, Turkey.
}

\begin{abstract}
Background. Chronic sinusitis and its complications are common in patients with cystic fibrosis. Mucoceles are one of these complications and can have life-threatening consequences if left untreated.

Case. We present the case of a giant ethmoid mucocele leading to proptosis and hypertelorism in a 5-year-old child with cystic fibrosis.

Conclusion. Chronic sinusitis and its complications are common in patients with CF. Mucoceles are a rare complication of sinusitis that can be treated surgically. As seen in this case if left untreated mucoceles can lead to orbital pathologies such as proptosis, hypertelorism. To the best of our knowledge, we report the first case report of giant ethmoidal mucocele leading to proptosis and hypertelorism in a patient with cystic fibrosis
\end{abstract}

Key words: cystic fibrosis, proptosis, mucocele, chronic sinusitis, endoscopic sinus surgery.

The prevalence of sinonasal pathology in patients with Cystic Fibrosis (CF) is extremely high. Due to the defect in Cystic Fibrosis TransmembraneConductance Regulator(CFTR) protein, stasis of viscous mucous and impaired mucociliary transport are believed to contribute to the development of sinonasal pathology. ${ }^{1}$ In general, CF patients exhibit both, chronic inflammation of the lower respiratory tract as well as chronic rhinosinusitis (CRS). Almost $100 \%$ of CF patients exhibit morphological changes in computed tomography (CT) of the nose and paranasal sinuses. Furthermore, it was shown, that the prevalence of rhinosinusitis is up to $63 \%$ and the prevalence of nasal polyps is $50 \%$ in adult CF patients. Only $7.1 \%$ of CF patients are free from inflammatory changes in sinonasal histology. ${ }^{2}$ Nasal polyps can be seen

$\triangle$ Orhan Tunç

orhantunc@gantep.edu.tr

Received 27th November 2019, revised 19th January 2020, accepted 27th January 2020. in up to $86 \%$ of children with $\mathrm{CF}$, but only in $6 \%$ of those under 6 years of age. Those with the $\Delta \mathrm{F} 508$ mutation are also more prone to have polypoidal manifestation. ${ }^{3}$ Mucoceles are a well-known complication of sinusitis in adults but they very rarely occur in the pediatric age. Mucoceles are secreting cysts lined with upper respiratory epithelium which can grow in the paranasal sinuses with a slow concentric expansion. They are benign, but can enlarge by accumulation of secretions, and may displace and destroy or erode the surrounding bone with local, orbital or even intracranial complications. ${ }^{4}$

Herein we present a 5-year-old patient with bilateral giant ethmoidal mucoceles causing proptosis and hypertelorism.

\section{Case Report}

A 5-year-old boy with CF was referred from the department of pediatrics to the otolaryngology department for nasal obstruction. He was the seventh child of a consanguineous family, 
and his two brothers had previously died for unknown reasons. The patient was followedup for recurrent lung infection at 5 months of age and $\triangle F 508$ mutations were detected and $C F$ was diagnosed. The patient's complaints were long-standing nasal congestion, runny nose, and snoring.

A standard ears nose throat (ENT) examination was performed and an endoscopic evaluation of the nose was performed. There was intense mucoid secretion in the nasal cavity and both inferior and middle turbinates were highly edematous. Nasal passage was completely closed by the inferior and middle turbinates. The patient had proptosis and hypertelorism in both eyes. Paranasal sinus tomography showed giant ethmoidal mucoceles causing propitosis and hypertelorism by pushing the orbita laterally (Fig. 1).

Functional endoscopic sinus surgery (FESS) was performed using a $0^{0}$ view, $2.7 \mathrm{~mm}$ rigid endoscopetoremove the masses and decompress the sinuses. The mucocele, which pushes the middle turbinate medially, was drained and the yellow-green colored viscous discharge was drained through the mucocele (Fig. 2). Although anterior and posterior ethmoidal cells did not complete their development, they were completely opened. Sphenoid sinus was not fully developed and surgery was not performed on the sphenoid sinus. Maxillary sinus ostium was enlarged. The wall of the cyst was completely removed and the mucosa on the lamina paprisea was preserved. Samples from mucocele and the discharge were sent for microbiology and histological assessments. Pseudomonas aeruginosa was observed in microbiological investigations. Although it was too early two months after surgery, there was a mild improvement in propitosis and

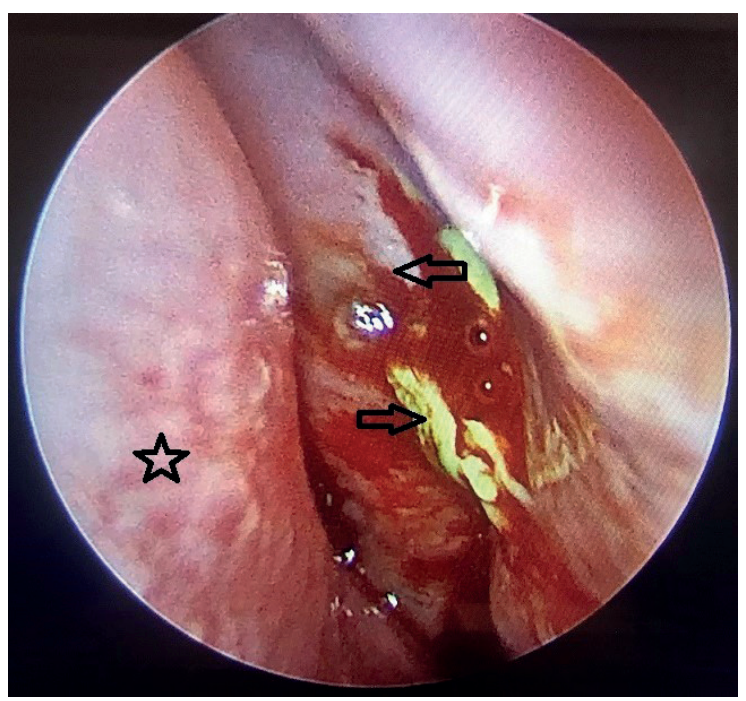

Fig. 2. Surgical image of left nasal cavity, (Black star; nasal septum, Right arrow; yellow green discharge, Left arrow; anterior wall of mucocele).
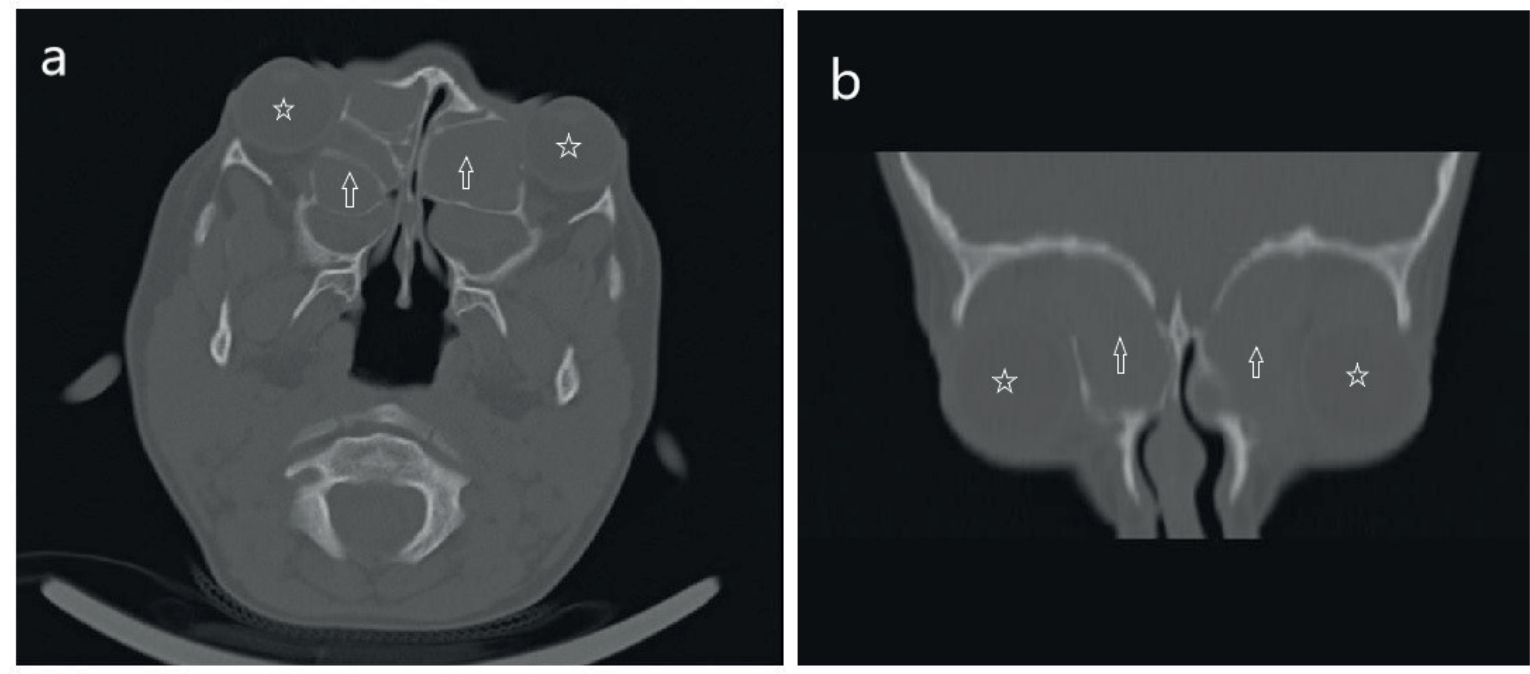

Fig. 1. (a) axial section of paranasal tomography, (b) coronal section of paranasal tomography (White stars; Approximate central alignment of the eye, White arrows; giant ethmoidal mucoceles). 
heipertelorism. The parents of the patient were informed about the operation and informed consent form was obtained so that it could be written as a case report.

\section{Discussion}

Infectious rhinosinusitis is a common disease in children; secondary bacterial infection or CRS are common complications, particularly in children affected by $\mathrm{CF}$, who generally harbour a large number of bacteria in sputum and nasal secretions. ${ }^{4}$ Sinus mucoceles are common complications of CRS in adults but are rare in the pediatric population, and no prevalence data currently exist for children with CF. Although an association is known between the two conditions, mucoceles are rarely the presenting feature of the underlying disease. Frontal sinus is most commonly affected by mucoceles and is involved in approximately two-thirds of cases, the ethmoids are the next most common site, and the maxillary and sphenoidal sinuses are less likely to be involved. ${ }^{5}$ Paranasal mucoceles leading to erosion of the surrounding bone walls was more frequent than expected. As the mucoceles expand, they distort local anatomy and apply pressure to surrounding structures. In patients with $\mathrm{CF}$, mucoceles may grow too large and cause orbital symptoms such as proptosis and hypertelorism. ${ }^{4}$

Pathogenesis of mucoceles is still not well understood in CF. Many factors may be involved including the degree of viscosity of the CF mucus, decreased mucociliary clearance due to low mucus hidratation or loss of ciliated cells or the presence of bacterially induced factors. Additionally, some anatomical predisposing factors limiting the drainage of ostium meatal complex may play a pathogenetic role and it may be suggested that in cases with a diagnosis during the initial months of life, the formation of the mucocele had already began in intrauterine life..$^{4,5}$

The pathophysiology for nasal polyp formation in CF is different from the non-CF population. Histologically, nasal polyps in CF consist of dilated mucous glandular structures, lack of eosinophils and a higher volume of neutrophils, mast cells and plasma cells. Microscopically, persistent bacteria colonization is the reason for a prolonged inflammatory response in the sinuses of patients with CF rather than the common viral vector. Pseudomonas aeruginosa is the commonest occupant, with a $95 \%$ prevalence rate. ${ }^{3}$

Current treatment involves FESS for drainage and marsupialisation of the mucocele. Topical steroid application is able to decrease the polyp size, but has limited effect on nasal symptoms due to the neutrophilic drive in CF nasal polyps. It seems performing FESS early in a child does not have any detrimental effect on the craniofacial development as first thought. Reviews have also shown a good safety profile for FESS in patients with CF. FESS relieves nasal symptoms, decreases hospitalization and assists with Pseudomonas aeruginosa eradication. ${ }^{3}$

Chronic sinusitis and its complications are common in patients with CF. Mucoceles are a rare complication of sinusitis that can be treated surgically. Untreated mucoceles can lead to orbital pathologies such as proptosis, hypertelorism.

To the best of our knowledge, we report the first case report of giant ethmoidal mucocele leading to proptosis and hypertelorism in a patient with $\mathrm{CF}$. Our recommendation to clinicians is to closely monitor patients with $\mathrm{CF}$ on nasal pathologies and to prevent possible complications.

\section{REFERENCES}

1. Berkhout MC, Klerx-Melis F, Fokkens WJ, Nuijsink M, van Aalderen WM, Heijerman HG. CT-abnormalities, bacteriology and symptoms of sinonasal disease in children with cystic fibrosis. J Cyst Fibros 2016; 15: 816-824.

2. Mainz JG, Schumacher U, Schädlich $K$, et al; Cooperators. Sino nasal inhalation of isotonic versus hypertonic saline $(6.0 \%)$ in CF patients with chronic rhinosinusitis-Results of a multicenter, prospective, randomized, double-blind, controlled trial. J Cyst Fibros 2016; 15: e57-e66. 
3. Mohd Slim MA, Dick D, Trimble K, McKee G. Paediatric nasal polyps in cystic fibrosis. BMJ Case Rep 2016; 2016: 214467.

4. Di Cicco M, Costantini D, Padoan R, Colombo C. Paranasal mucoceles in children with cystic fibrosis. Int J Pediatr Otorhinolaryngol 2005; 69: 1407-1413.
5. Qureishi A, Lennox P, Bottrill I. Bilateral maxillary mucoceles: an unusual presentation of cystic fibrosis. J Laryngol Otol 2012; 126: 319-321. 